\title{
Computational Modeling of Ultrasonic Subthalamic Nucleus Stimulation
}

\author{
Thomas Tarnaud, Wout Joseph, Luc Martens, Emmeric Tanghe
}

\begin{abstract}
Objective: To explore the potential of ultrasonic modulation of plateau-potential generating subthalamic nucleus neurons (STN), by modeling their interaction with continuous and pulsed ultrasonic waves. Methods: A computational model for ultrasonic stimulation of the STN is created by combining the Otsuka-model with the bilayer sonophore model. The neuronal response to continuous and pulsed ultrasonic waves is computed in parallel for a range of frequencies, duty cycles, pulse repetition frequencies, and intensities. Results: Ultrasonic intensity in continuous-wave stimulation determines the firing pattern of the STN. Three observed spiking modes in order of increasing intensity are low frequency spiking, high frequency spiking with significant spike-frequency and spike-amplitude adaptation, and a silenced mode. Continuous-wave stimulation has little capability to manipulate the saturated spiking rate in the high frequency spiking mode. In contrast, STN firing rates induced by pulsed ultrasound insonication will saturate to the pulse repetition frequency with short latencies, for sufficiently large intensity and repetition frequency. Conclusion: Computational results show that the activity of plateau-potential generating subthalamic nucleus neurons can be modulated by selection of the stimulus parameters. Low intensities result in repetitive firing, while higher intensities silence the STN. Pulsed ultrasonic stimulation results in a shorter saturation latency and is able to modulate spiking rates. Significance: Stimulation or suppresion of the STN is important in the treatment of Parkinson's disease, e.g., in deep brain stimulation. This explorative study on ultrasonic modulation of the STN, could be a step in the direction of minimally invasive alternatives to conventional deep brain stimulation.
\end{abstract}

Index Terms-Acoustical neurostimulation, Computational modeling, Otsuka-model, Subthalamic nucleus (STN), ultrasonic neuromodulation

This work was carried out using the Supercomputer Infrastructure (STEVIN) at Ghent University, funded by Ghent University, the Flemish Supercomputer Center (VSC), the Hercules Foundation and the Flemish Government department EWI.

This research was funded by the FWO-project G046816N. T. Tarnaud is a PhD Fellow of the FWO-V (SB) (Research Foundation Flanders, Belgium). E. Tanghe is a Post-Doctoral Fellow of the FWO-V (Research Foundation Flanders, Belgium)

T. Tarnaud, W. Joseph, L. Martens, and E. Tanghe are with the Department of Information Technology (INTEC-WAVES/IMEC), Ghen University/IMEC, Technologypark 15, 9052 Zwijnaarde, Belgium. E-mail: thomas.tarnaud@ugent.be

Manuscript received ; revised

\section{INTRODUCTION}

UBTHALAMIC nucleus deep brain stimulation (STN$\checkmark$ DBS) is a surgical treatment that is mainly used to treat symptoms (rigidity, bradykinesia, tremor, and levodopainduced dyskinesias) in patients with advanced Parkinson's disease [1]. However, although DBS has proven to be effective in appropriately selected patients, the surgery is associated with significant risks, such as haemmorhage and infection [2], [3]. As a consequence, several authors have explored the possibility of non-invasive alternatives to conventional deep brain stimulation. Two examples are temporal-interference and wireless magnetothermal deep brain stimulation [4]-[6].

Another option is the use of ultrasound (US) to reversibly modulate neuronal activity. As multiple-element phased-arrays of ultrasound transducers have been used for non-invasive thalamotomy or tumour ablation with millimeter precision [7][10], the possibility of using similar array designs for ultrasonic modulation of deep brain structures has been considered before [4], [11], [12]. Although the initial idea of using ultrasound to modulate neuronal activity is almost nine decades old [13], [14], the field has recently gained significant attention [11], [12], [15]-[23]. In conclusion, transcranial focused ultrasound seems to be promising as alternative for conventional deep brain stimulation, because of its theoretical ability to modulate deep brain nuclei non-invasively, selectively and with high-precision and without the need to genetically alter the target neurons (cfr. wireless magnetothermal DBS and optogenetics).

However, the therapeutical potential of ultrasonic subthalamic nucleus stimulation is still elusive, as no experimental or computational studies have been performed on this topic. The goal of this paper is to provide for the first time an explorative and computational study on ultrasonic stimulation of subthalamic nucleus neurons.

Although the nature of this study is completely computational and experimental validation will be necessary, we believe that at this stage a theoretical investigation on the potential of ultrasonic STN-stimulation is worthwhile, because:

- Interpretation of US-STN stimulation might not be straightforward without computational modeling, due to the ability of subthalamic nucleus neurons to generate plateau-potentials in a voltage-dependent manner [24][27]. Furthermore, the mechanism of ultrasonic neuromodulation is likely to be indirect, mediated by membrane charge accumulation driven by the hyperpolarized state of the neuronal membrane [28]-[30]. As a consequence, the relation between the stimulation param- 
eters (frequency, intensity, duty cycle, pulse-repetition frequency...) and the observed neuronal behaviour, is less intuitive for ultrasonic stimulation than for electrical stimulation. Here, computational modeling might help to obtain a clear understanding of how these stimulation parameters interact with the intrinsic dynamics of plateaupotential generating STN-neurons. In this regard, comparison of the presented computational results with future experimental studies might improve understanding on the underlying mechanism of ultrasonic neuromodulation.

- We constructed a computational model of ultrasonic subthalamic nucleus stimulation, from the Otsuka-model of plateau-potential generating STN-neurons [26] and the bilayer sonophore model of ultrasound-membrane coupling [29] (BLS-model). Although no experimental data on ultrasonic STN stimulation exists, several experimental studies correspond well with both the Otsuka-model and the BLS-model.

- The presented results can be used as a basis for future computational work in which the impact of ultrasound on the basal ganglia system is investigated, paving the way for in vivo validation. Futhermore, this study might inspire future translational bioengineering efforts, focusing on the design of the transducer arrays required for non-invasive ultrasonic deep brain stimulation.

\section{Methods}

The dynamics of the neuronal membrane as a bilipid layer in the presence of an ultrasonic source is modeled by the bilayer sonophore (BLS) model [28]. In this model, a modified Rayleigh-Plesset equation is used to calculate the maximal displacement $Z$ of the membrane with respect to its resting position:

$$
\begin{gathered}
\frac{\mathrm{d}^{2} Z}{\mathrm{~d} t^{2}}+\frac{3}{2 R(Z)}\left(\frac{\mathrm{d} Z}{\mathrm{~d} t}\right)^{2}=\frac{1}{\rho_{\mathrm{l}}|R(Z)|}\left[P_{\mathrm{in}}+P_{\mathrm{M}}+P_{\mathrm{ec}}-P_{0}+\right. \\
\left.P_{\mathrm{A}} \sin (\omega t)-P_{\mathrm{S}}(Z)-\frac{4}{|R(Z)|} \frac{\mathrm{d} Z}{\mathrm{~d} t}\left(\frac{3 \delta_{0} \mu_{\mathrm{s}}}{|R(Z)|}+\mu_{\mathrm{l}}\right)\right] .
\end{gathered}
$$

Here, $R(Z)=\left(a^{2}+Z^{2}\right) /(2 Z)$ is the radius of curvature of a leaflet of the symmetrical bilipid layer, $\rho_{\mathrm{l}}=1028 \frac{\mathrm{kg}}{\mathrm{m}^{3}}$ is the density of the surrounding liquid $(a=32 \mathrm{~nm}$ is the radius of the considered circular membrane patch), and $\omega=2 \pi f$ is the angular frequency of the US-wave. The pressure terms $P_{\mathrm{in}}, P_{\mathrm{M}}, P_{\mathrm{ec}}, P_{0}, P_{\mathrm{A}}$, and $P_{\mathrm{S}}(Z)$ represent the internal gas pressure, molecular pressure, electrostatic pressure, static pressure, amplitude of the applied ultrasonic source, and the pressure due to the tension in the leaflets, respectively. The last term in (1) represents the effect of viscosity, with $\delta_{0}$ the thickness of a leaflet, $\mu_{\mathrm{s}}$ the dynamic viscosity of the leaflet and $\mu_{1}$ is the dynamic viscosity of the cerebrospinal fluid (CSF).

In the neuronal intramembrane cavitation excitation (NICE) model, the dynamics of the bilipid layer (eq. (1) is coupled with a Hodgkin-Huxley type equation, by adding a displacement current $V \frac{\mathrm{d} C_{\mathrm{m}}}{\mathrm{d} t}$ [29], [30]. Here, $C_{\mathrm{m}}$ is a function of the displacement $Z$ :

$$
C_{\mathrm{m}}(Z)=\frac{C_{\mathrm{m} 0} \Delta}{a^{2}}\left(Z+\frac{a^{2}-Z^{2}-Z \Delta}{2 Z} \ln \left(\frac{2 Z+\Delta}{\Delta}\right)\right) .
$$

$C_{\mathrm{m} 0}=1 \mu \mathrm{F} / \mathrm{cm}^{2}$ is the membrane capacitance of the bilipid layer at rest $\left(P_{\mathrm{A}}=0 \mathrm{~Pa}\right)$ and $\Delta=1.29 \mathrm{~nm}$ is the separation between the leaflets if the bilipid layer is at equilibrium (calculated for $V=-58 \mathrm{mV}$ ). Equations for the mentioned pressure terms, as well as model constants of the BLS-model, are adopted from [28]-[30] and are summarized in Appendix A.

In this study, ultrasonic neuromodulation of the plateaupotential generating subthalamic nucleus neuron is modeled by adding the capacitive displacement current $I_{\mathrm{D}}=V \frac{\mathrm{d} C_{\mathrm{m}}}{\mathrm{d} t}$ to the Otsuka-model [26], [29], [31]:

$$
C_{\mathrm{m}} \frac{\mathrm{d} V}{\mathrm{~d} t}+V \frac{\mathrm{d} C_{\mathrm{m}}}{\mathrm{d} t}=-I_{\mathrm{Na}}-I_{\mathrm{K}}-I_{\mathrm{A}}-I_{\mathrm{T}}-I_{\mathrm{L}}-I_{\mathrm{CaK}}-I_{\mathrm{l}} .
$$

Here, $C_{\mathrm{m}}$ is the membrane capacitance and $V$ is the membrane potential. The model includes a sodium current $\left(I_{\mathrm{Na}}\right)$, a delayed-rectifier $\mathrm{K}^{+}$current $\left(I_{\mathrm{K}}\right)$, an A-type $\mathrm{K}^{+}$current $\left(I_{\mathrm{A}}\right)$, a low threshold $\mathrm{Ca}^{2+}$ current $\left(I_{\mathrm{T}}\right)$, a high-threshold $\mathrm{Ca}^{2+}$ current $\left(I_{\mathrm{L}}\right)$, a $\mathrm{Ca}^{2+}$ dependent $\mathrm{K}^{+}$current $\left(I_{\mathrm{CaK}}\right)$ and a leak current $\left(I_{1}\right)$. Equations for the membrane currents and gate parameters and the associated constants are adopted from Otsuka et al. and summarized in Appendix A [26].

The calcium dynamics were modeled as in Otsuka [26] and Hahn and McIntyre [32]:

$$
\frac{\mathrm{d} C a_{\mathrm{i}}}{\mathrm{d} t}=-\alpha\left(I_{\mathrm{T}}+I_{\mathrm{L}}\right)-K_{\mathrm{Ca}} C a_{\mathrm{i}},
$$

where $C a_{\mathrm{i}}$ is the calcium concentration available near the calcium-dependent membrane gates. The intracellullar calcium concentration is increased by calcium currents $\left(I_{\mathrm{T}}\right.$ and $\left.I_{\mathrm{L}}\right)$ and decreased by calcium pumps $\left(K_{\mathrm{Ca}}=2 \mathrm{~ms}^{-1}\right)$. Finally, $\alpha=1 /\left(2 F d_{\text {eff }}\right)$, with $F$ the Faraday constant and $d_{\text {eff }}$ an effective depth, accounting for buffering mechanisms and geometry considerations 1 . In this study, $d_{\text {eff }}$ is calculated so that the equilibrium calcium concentration is $5 \mathrm{nM}$ at $V=-58 \mathrm{mV}$ and $T=33{ }^{\circ} \mathrm{C}$.

\section{Spike rate, latency and saturation intensity}

Incidence of a neuronal spike is determined by the zerocrossing with positive slope of the membrane charge $Q=$ $C_{m} V$. The spiking time $t_{i}^{\mathrm{AP}}$ corresponds with the $i$ th spike after ultrasonic stimulus onset. The interspike interval (ISI) is the time interval between two subsequent spikes: ISI $_{i}=$ $t_{i+1}^{\mathrm{AP}}-t_{i}^{\mathrm{AP}}$. An instantaneous spiking rate $\mathrm{SR}_{i}$ is then defined as the reciprocal of the interspike interval (i.e., $\mathrm{SR}_{i}=\mathrm{ISI}_{i}^{-1}$ ). In spiking rate plots, $\mathrm{SR}_{i}$ is plotted against the spiking times $t_{i}^{\mathrm{AP}}$.

Spiking rates of the subthalamic nucleus, insonicated with a pulsed wave with sufficiently high pulse repetition frequency $(\mathrm{PRF} \geq 100 \mathrm{~Hz})$ and ultrasonic intensity $\left(I_{\mathrm{US}}>I_{\mathrm{sat}}\right)$, will saturate to the pulse repetition frequency (i.e., $\exists i_{\text {sat }}, \forall j \geq$ $\left.i_{\mathrm{sat}}:\left|\mathrm{SR}_{j}-\mathrm{PRF}\right| \leq \alpha_{\mathrm{c}} \mathrm{PRF}\right)$. Here $I_{\text {sat }}$ is the saturation

${ }^{1}$ Note that although $d_{\text {eff }}$ is not mentioned in Otsuka et al., it has to be included in the definition of $\alpha$ for dimensional consistency 

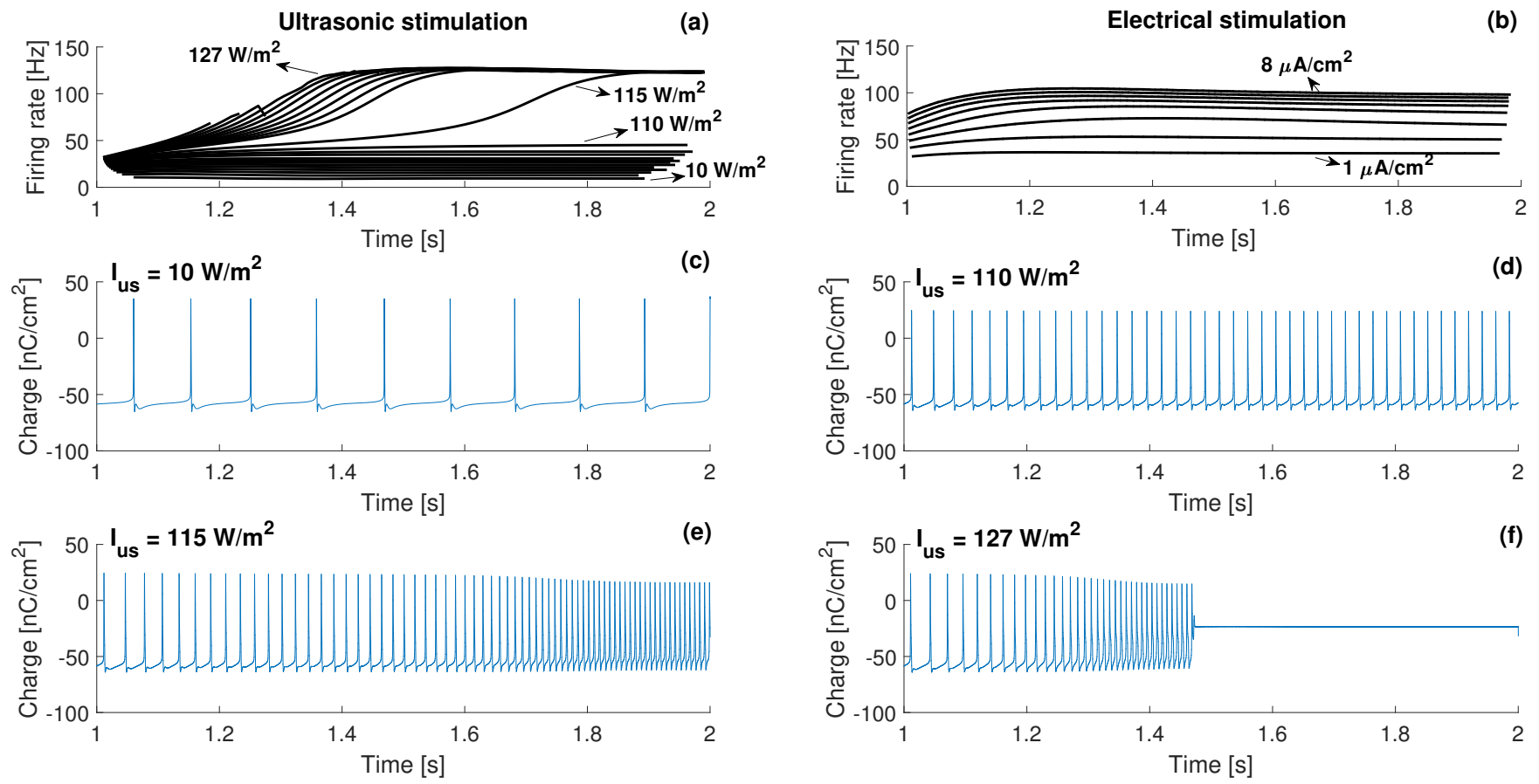

Fig. 1 (a)-(b) Spike firing rate as function of time after onset of stimulation (pulse start PS $=1$ s, pulse duration PD $=1 \mathrm{~s}$, $f=0.69 \mathrm{MHz}$ ). (a) Ultrasonic continuous-wave stimulation of Otsuka-STN model. (b) Electrical stimulation of Otsuka-STN model. (c-f) Membrane charge versus time for the ultrasonic stimulation configurations indicated with arrows in (a).

intensity and $\alpha_{\mathrm{c}}$ is a convergence factor. The latency is then defined as the corresponding spiking time $t_{i_{\mathrm{sat}}}^{\mathrm{AP}}$. For continuouswave stimulation, the saturated spiking rate is defined as the inverse of the interspike interval at the end of the ultrasonic stimulus.

In this study, all ultrasonic intensities are reported in their spatial-peak pulse-averaged (sppa) form and are related with the applied ultrasonic pressure $P_{\mathrm{A}}$ by:

$$
I_{\text {sppa }}=\frac{P_{\mathrm{A}}^{2}}{2 \rho_{\mathrm{l}} c} .
$$

Here, $c$ represents the speed of sound.

\section{RESULTS}

\section{A. Model verification ${ }^{2}$}

In this study, a model for ultrasonic stimulation of the subthalamic nucleus was constructed, by coupling a HodgkinHuxley type model (Otsuka-model of the STN, [26]) with the bilayer sonophore model [28] of ultrasound-membrane interaction. Although this is the first modeling study of ultrasonic STN-stimulation, partial verification of our implementation of the BLS-STN model was still possible, by closely comparing results with literature [29]-[31]. More specifically, we verified the implementation of the Otsuka-model without ultrasonic stimulation $\left(P_{\mathrm{A}}=0 \mathrm{~Pa}\right)$ by reproducing the observed features of the STN-model (spontaneous firing, steady state I-V

\footnotetext{
${ }^{2} \mathrm{~A}$ distinction between verification and validation is made in this study. Verification refers to the correctness of the implementation and numerical convergence. Validation refers to the correspondence with experimental results.
}

curves, plateau-potential generation, dependency of firing rate on injected current, and rebound spiking) [31]. In order to verify our implementation of the bilayer sonophore model, we reproduced the results obtained by Plaksin et al. [29], [30], for ultrasonic stimulation of cortical (RS, FS, and LTS) neurons (NICE-model).

The model equations are solved in a similar fashion to Plaksin et al. [29], [30]. Eq. (1) and eq. (3) are solved by separate ode113-Matlab functions. The ode113-solver is a variable step variable order method, which uses AdamsBashforth-Moulton predictor-corrector pairs of variable order between 1 and 13 [33], [34]. The membrane charge in eq. (1) is updated every $T_{\mathrm{up}}=25 \mu \mathrm{s}$ (we observed that a shorter update time $T_{\text {up }}$ was required for simulations with the OtsukaSTN model, as compared to simulations with cortical RS, FS and LTS neurons [29], [30]). A maximal step size of $\mathrm{d} t=0.025 / f$, as well as appropriate absolute and relative tolerances are imposed on the ode113-solvers. To ensure that the results are independent of the implementation details, control simulations were performed with a smaller maximal timestep $(\mathrm{d} t=0.0125 / f)$, smaller update time $\left(T_{\mathrm{up}}=12.5 \mu \mathrm{s}\right)$, smaller absolute and relative tolerances (factor 10) and higher resolution for the look-up tables of the rate constants (refinement factor 10). The mentioned discretization parameters were determined by refinement until relative errors, obtained by comparison of the default and control simulations for continuous-wave stimulation, are lower than $10 \%$.

In Fig. 1 the spiking modes of the subthalamic nucleus, due to continuous-wave ultrasonic stimulation are summarized and 


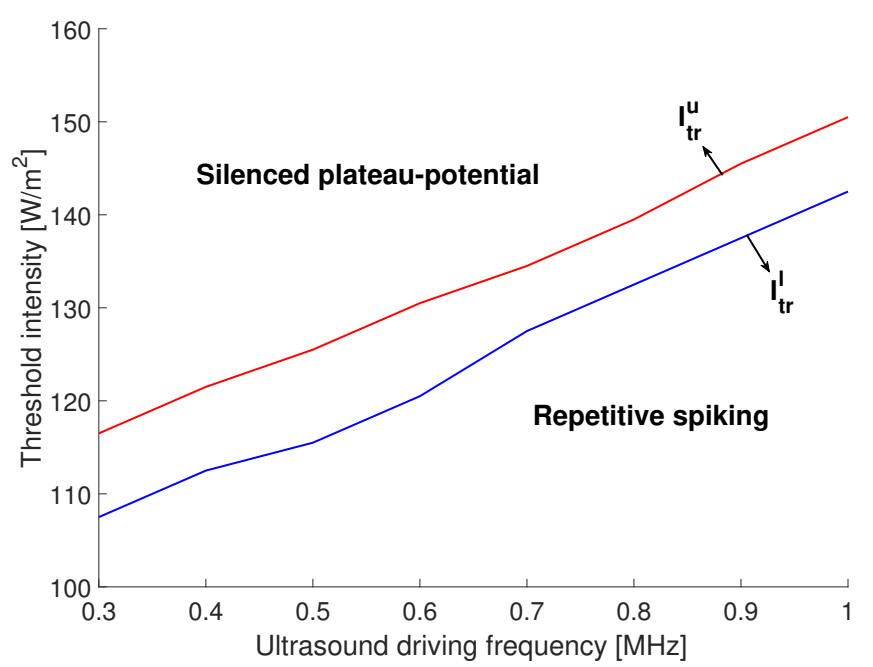

Fig. 2 Upper and lower threshold intensity $I_{\mathrm{tr}}^{\mathrm{u}}$ and $I_{\mathrm{tr}}^{\mathrm{l}}$ versus ultrasonic driving frequency. Low ultrasonic intensities $(I<$ $\left.I_{\mathrm{tr}}^{\mathrm{l}}\right)$ result in repetitive firing, while high intensities $\left(I>I_{\mathrm{tr}}^{\mathrm{u}}\right)$ will generate silenced plateaus. The intermediate regime $\left(I_{\mathrm{tr}}^{1}<\right.$ $\left.I<I_{\mathrm{tr}}^{\mathrm{u}}\right)$ corresponds with STN-neurons, that will fire some spikes at stimulation onset, before transitioning to a stable plateau. Threshold are determined with $\mathrm{PD}=1 \mathrm{~s}$.

a comparison is made between ultrasonically and electrically induced firing rate curves. The ultrasonic firing rate curves in Fig. 11a) are used to determine numerical convergence, by calculation of the relative error on the maximal and saturated firing rate $\left(f_{\max }\right.$ and $\left.f_{\text {sat }}\right)$ and the peak firing rate time $\tau_{\mathrm{p}}$. Because a change in neuronal spiking rythm can be observed when increasing the ultrasonic intensity from $110 \mathrm{~W} / \mathrm{m}^{2}$ to $115 \mathrm{~W} / \mathrm{m}^{2}$ (Fig. 1 (a)), relative errors are calculated separately for low intensities $\left(10 \mathrm{~W} / \mathrm{m}^{2}\right.$ to $100 \mathrm{~W} / \mathrm{m}^{2}$, sampling step of $10 \mathrm{~W} / \mathrm{m}^{2}$ ) and intermediate intensities (from $120 \mathrm{~W} / \mathrm{m}^{2}$ to the lower intensity threshold $I_{\mathrm{tr}}^{\mathrm{l}}$, see section III-B sampling step of $\left.1 \mathrm{~W} / \mathrm{m}^{2}\right)^{3}$. Comparison between the default and control simulations indicates that relative errors are always lower than $10 \%$. In particular, maximal errors are $7.72 \%$, $6.94 \%, 9.9844 \%$ (low intensities) and $0.59 \%, 1.47 \%, 3.14 \%$ (intermediate intensities) for the saturated firing rate, maximal firing rate, and peak firing rate time, respectively. The relative error on the low intensity threshold $I_{\mathrm{tr}}^{\mathrm{l}}$ (see section III-B) is lower than $1 \%$.

B. Continuous-wave stimulation: excitation by low-intensity, silencing by high-intensity ultrasound

A first prediction obtained from the BLS-STN model is that stimulation of a plateau-potential generating STN-neuron with a continuous-wave of low-intensity ultrasound leads to a sequence of action potentials, while higher intensities will silence the STN-neuron by generation of a plateau-potential.

\footnotetext{
${ }^{3}$ In this study, only neuromodulation by low-intensity focused ultrasound (LIFU) is considered. For convenience, we use the relative terms low, intermediate and high intensity to refer to the intensities, corresponding with the observed spiking modes.
}

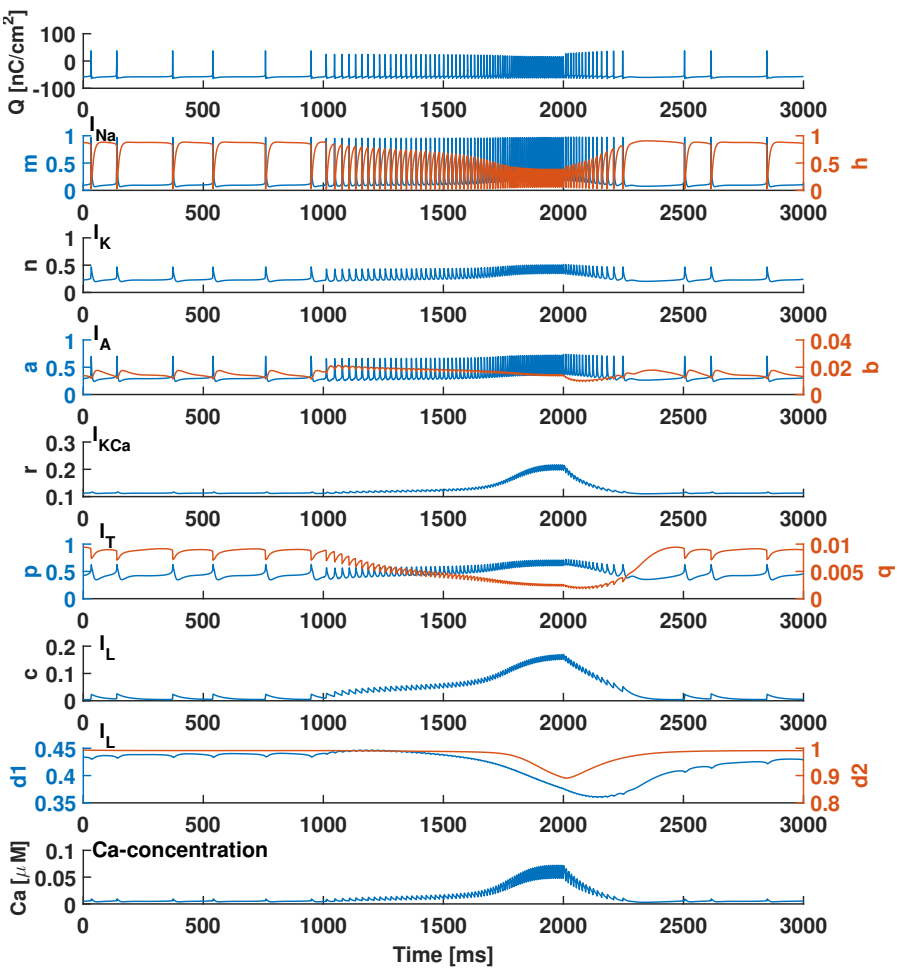

Fig. 3 Membrane channel dynamics of Otsuka-STN model, stimulated by low intensity ultrasound $\left(I=115 \mathrm{~W} / \mathrm{m}^{2}\right)$. The traces represent the membrane charge $Q$, the intracellullar calcium concentration $C a_{\mathrm{i}}$ and the gate parameters $\left(I_{\mathrm{Na}}(m\right.$, $\left.h), I_{\mathrm{K}}(n), I_{\mathrm{T}}(p, q), I_{\mathrm{KCa}}(r), I_{\mathrm{A}}(a, b), I_{\mathrm{L}}\left(c, d_{1}, d_{2}\right)\right)$.

This is illustrated in Fig. 11(a) ( $f=0.69 \mathrm{MHz}$ sonication of BLS-STN model). Low ultrasonic intensities $\left(I_{\mathrm{US}} \leq\right.$ $110 \mathrm{~W} / \mathrm{m}^{2}$ ) modulate the behaviour of the subthalamic nucleus by inducing repetitive spiking at low rates $(\leq 45 \mathrm{~Hz})$ and with small spike-frequency adaptation. Furthermore, the amplitude of the spikes induced by low ultrasonic intensities is approximately constant during sonication (Fig. 1 (c-d)). Increasing the ultrasonic intensity $\left(115 \mathrm{~W} / \mathrm{m}^{2} \leq I_{\mathrm{US}} \leq\right.$ $126 \mathrm{~W} / \mathrm{m}^{2}$ ) leads to high-frequency spiking with significant spike-frequency and amplitude adaptation during the pulse duration (Fig. 11(a) and Fig. 1(e)). For instance, for $I_{\mathrm{US}}=$ $115 \mathrm{~W} / \mathrm{m}^{2}$, the subthalamic nucleus will reach spiking rates of about $120 \mathrm{~Hz}$ (saturated spiking-frequency), after a spikefrequency adaptation period of about $850 \mathrm{~ms}$. For intensities high enough to induce high-frequency spiking with significant spike-frequency adaptation, increasing the ultrasonic intensity will not affect the saturated spiking rate (between $124 \mathrm{~Hz}$ and $128 \mathrm{~Hz}$, Fig. 17, but will shorten the timespan necessary to obtain a stabilized spiking rate (peak firing rate times decrease to $500 \mathrm{~ms}$, when increasing the ultrasonic intensity $I_{\mathrm{US}}$ to the lower intensity threshold $I_{\mathrm{tr}}^{1}=126.5 \mathrm{~W} / \mathrm{m}^{2}$ ).

Plateau-generating subthalamic nucleus neurons will be silenced at a stable plateau potential, if sonicated with a $0.69 \mathrm{MHz}$ continuous ultrasonic wave with intensity larger than $I_{\mathrm{tr}}^{1}=126.5 \mathrm{~W} / \mathrm{m}^{2}$ (Fig. 1 (a) and Fig. 1 f)). This stable plateau potential is preceded by a "transient regime" of repetitive spiking. With increasing ultrasonic intensity, the 

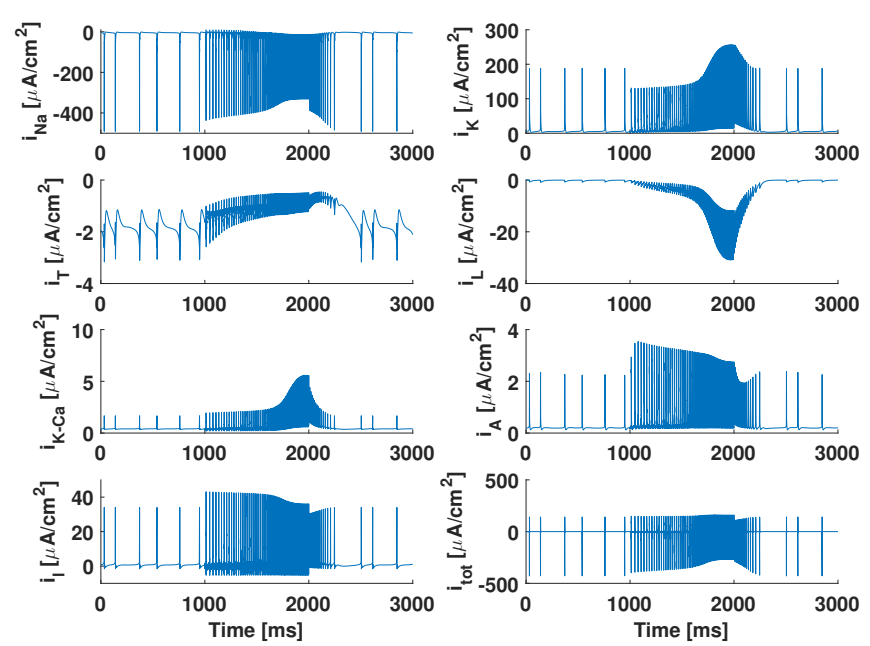

Fig. 4 Membrane channel dynamics of Otsuka-STN model, stimulated by low intensity ultrasound $\left(I=115 \mathrm{~W} / \mathrm{m}^{2}\right)$. The traces represent the active $\left(I_{\mathrm{Na}}, I_{\mathrm{K}}, I_{\mathrm{T}}, I_{\mathrm{L}}, I_{\mathrm{KCa}}\right.$, and $\left.I_{\mathrm{A}}\right)$ and passive $\left(I_{1}\right)$ membrane currents, as well as total current flowing through the active membrane $I_{\text {tot }}$.

duration of this transient regime decreases. Eventually, for ultrasonic intensities larger than $I_{\mathrm{tr}}^{\mathrm{u}}=135 \mathrm{~W} / \mathrm{m}^{2}$, the stable plateau potential induced by the ultrasonic wave will be preceded by a single action potential.

The results obtained for ultrasonic sonication of the Otsukamodel are compared with electrical stimulation results in Fig. 1 (b) (constant current injected in subthalamic nucleus model neurons). Spike-frequency adaptation is of course also present for electrical stimulation, but is much more modest than in ultrasonic stimulation. Furthermore, initial spiking frequency is higher in electrically stimulated than in ultrasonically stimulated neurons. Finally, no transition from repetitive spiking to a stable plateau for higher current injections is observed.

The impact of the frequency of the continuous ultrasonic wave on the transition from high-frequency spiking to a stable plateau-potential, is illustrated in Fig. 2 (lower and upper ultrasonic intensity thresholds $I_{\mathrm{tr}}^{\mathrm{l}}$ and $I_{\mathrm{tr}}^{\mathrm{u}}$ as function of frequency). Due to the inertia of the cerebrospinal fluid surrounding the oscillating intraleaflet cavity, higher frequencies will have higher threshold intensities. Fig. 2 defines a thresholdfrequency curve, delineating different neuronal spiking modes, useful for experimental validation of the BLS-STN model.

\section{High-threshold calcium current mediates spike-frequency adaptation. Steady plateaus are maintained by calcium cur- rents.}

The membrane channel dynamics responsible for the spikefrequency adapting high-frequency firing in $\mathrm{STN}$-neurons is shown in Fig. 3 The corresponding membrane currents are shown in Fig. 4 Here, the Otsuka-model is simulated for insonication with $I_{\mathrm{US}}=115 \mathrm{~W} / \mathrm{m}^{2}(f=0.69 \mathrm{MHz})$.

Most of the time-dependence after stimulus onset is due to the slowly decaying and depolarization activated $c$-parameter

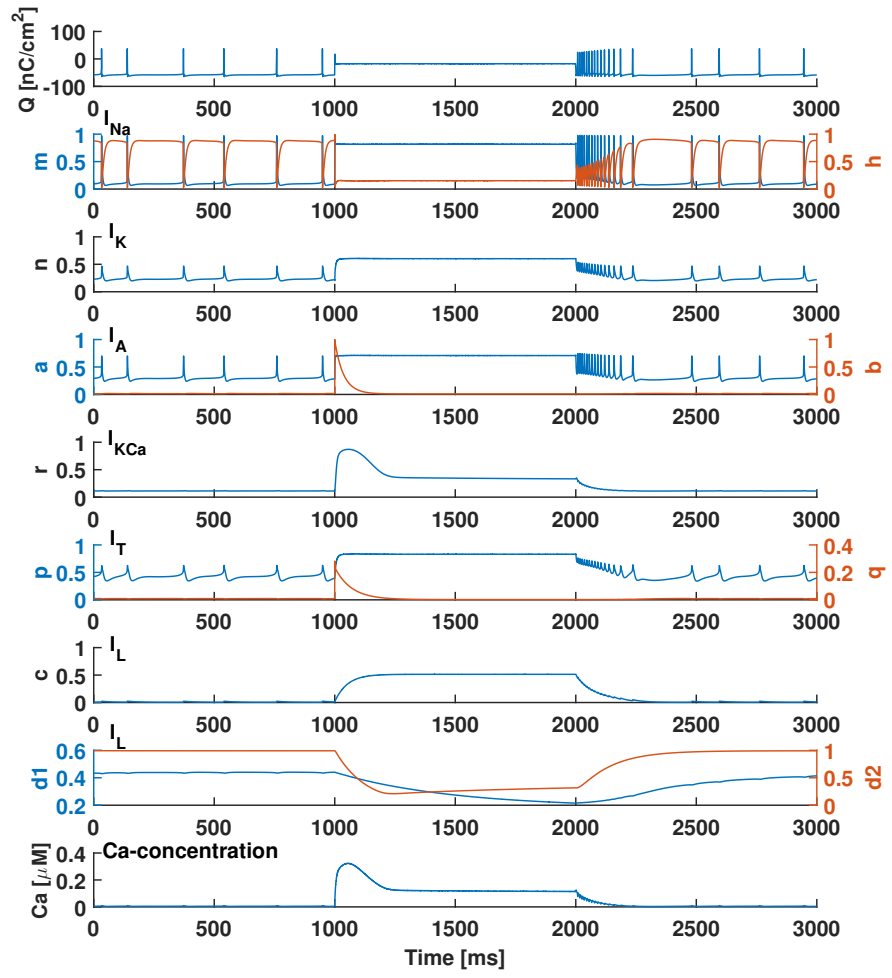

Fig. 5 Membrane channel dynamics of Otsuka-STN model, stimulated by high intensity ultrasound $\left(I=1000 \mathrm{~W} / \mathrm{m}^{2}\right)$. The traces represent the membrane charge $Q$, the intracellullar calcium concentration $C a_{\mathrm{i}}$ and the gate parameters $\left(I_{\mathrm{Na}}(m\right.$, $\left.h), I_{\mathrm{K}}(n), I_{\mathrm{T}}(p, q), I_{\mathrm{KCa}}(r), I_{\mathrm{A}}(a, b), I_{\mathrm{L}}\left(c, d_{1}, d_{2}\right)\right)$.

of the high-threshold calcium current $I_{\mathrm{L}}$. Blocking the $I_{\mathrm{L}}$ current in the Otsuka-model $\left(g_{\mathrm{L}}=0 \mathrm{~S} / \mathrm{m}^{2}\right)$, indeed shows disruption of the spike-frequency and spike-amplitude adaptation, which is replaced by steady and low frequency firing at rates of about $23 \mathrm{~Hz}$ (results not shown).

The results on the membrane dynamics of repetitively firing STN-neurons (Fig. 3 and Fig. 4) should be compared with the membrane parameters and currents, obtained for silenced STN-neurons (insonication with $I=1000 \mathrm{~W} / \mathrm{m}^{2}$ $(f=0.69 \mathrm{MHz}))$ in Fig. 5 and Fig. 6 , respectively.

The membrane gate parameters will reach steady values, some time after the plateau potential has been reached. The action potential that precedes the plateau potential is generated by a short ultrasound-induced hyperpolarization. This hyperpolarization is a consequence of the oscillating membrane capacitance (membrane potential not shown). Note that this short ultrasound-driven hyperpolarization before the action potential preceding the plateau-potential is strong enough to deinactive the membrane gates $b$ and $q$ at stimulus onset. This results in a short peaktime of the A-type K-current $I_{\mathrm{A}}$ and the T-type (low threshold) Ca-current $I_{\mathrm{T}}$ (Fig.6. In contrast, smaller ultrasonic intensities $I_{\mathrm{US}}$ (but still high enough to induce a plateau: $I_{\mathrm{US}}>I_{\mathrm{tr}}^{\mathrm{l}}$ ) are not able to deinactive these gate-parameters, resulting in smaller $I_{\mathrm{T}}$ and $I_{\mathrm{A}}$ values.

The calcium currents $I_{\mathrm{L}}$ and $I_{\mathrm{T}}$ prevent the membrane from repolarizing during the long plateau by injecting charge (i.e., depolarizing currents), while $I_{\mathrm{A}}, I_{\mathrm{KCa}}, I_{1}$ and $I_{\mathrm{Na}}+I_{\mathrm{K}}$ are ex- 

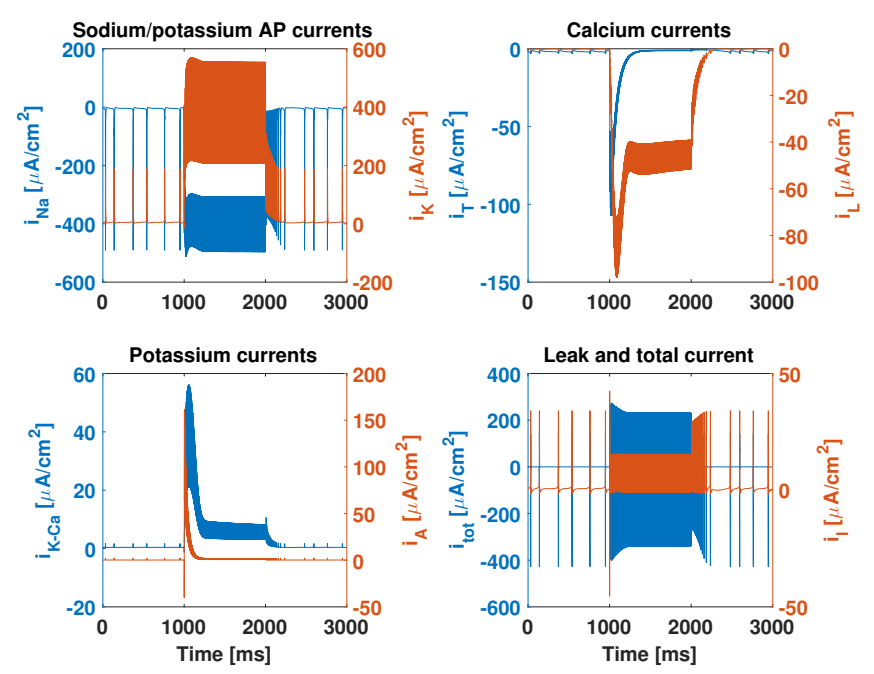

Fig. 6 Membrane channel dynamics of Otsuka-STN model, stimulated by high intensity ultrasound $\left(I=1000 \mathrm{~W} / \mathrm{m}^{2}\right)$. The traces represent the active $\left(I_{\mathrm{Na}}, I_{\mathrm{K}}, I_{\mathrm{T}}, I_{\mathrm{L}}, I_{\mathrm{KCa}}\right.$, and $\left.I_{\mathrm{A}}\right)$ and passive $\left(I_{1}\right)$ membrane currents, as well as total current flowing through the active membrane $I_{\text {tot }}$.

tracting charge from the membrane (i.e., repolarizing currents). However, closer observation of the injected charge indicates that in the first $20 \mathrm{~ms}$ after stimulus onset, the contribution of $I_{\mathrm{Na}}+I_{\mathrm{K}}$ to the total current is actually depolarizing, due to the mostly hyperpolarizing oscillations of the membrane potential in phase with the ultrasonic wave, while the contribution of $I_{\mathrm{L}}+I_{\mathrm{T}}+I_{\mathrm{A}}+I_{\mathrm{KCa}}$ is still repolarizing. Nevertheless, the relatively slow $n$-gate parameter will then saturate (about $20 \mathrm{~ms}$ after stimulus onset) and the $I_{\mathrm{Na}}+I_{\mathrm{K}}$-contribution becomes repolarizing. At the same time $I_{\mathrm{L}}+I_{\mathrm{T}}+I_{\mathrm{A}}+I_{\mathrm{KCa}}$ will start to inject positive charge. As we noted earlier, the relative contributions of $I_{\mathrm{T}}$ and $I_{\mathrm{L}}$ to the long plateau depends on the ultrasonic intensity of the incident wave. $I_{\mathrm{L}}$ becomes relatively more important for lower ultrasonic intensities, when the inactivation constant $q$ of the low-threshold calcium current $I_{\mathrm{T}}$ is not deinactivated anymore by the short ultrasoundinduced hyperpolarization at stimulus onset.

D. Pulse repetition frequency determines STN saturating firing rate. Latencies are reduced at high duty cycles and intensities.

At sufficiently high intensities $\left(I_{\mathrm{US}}>I_{\mathrm{sat}}\right)$ and pulse repetition frequencies $\left(\mathrm{PRF}>\mathrm{PRF}_{\min }>90 \mathrm{~Hz}\right)$, pulsed ultrasonic waves are able to entrain subthalamic nucleus spiking patterns. Here, we define the saturation intensity $I_{\text {sat }}$ as the minimal ultrasonic intensity for which the firing rate will saturate at the pulse repetition frequency. This is illustrated in Fig. 7(a),(c) for ultrasonic stimulation with $\mathrm{PRF}=100 \mathrm{~Hz}$, duty cycle $\mathrm{DC}=10 \%$, and intensity $I_{\mathrm{US}}=270 \mathrm{~W} / \mathrm{m}^{2}$. After a latency of $300 \mathrm{~ms}$ the spiking rate will saturate, i.e., $\operatorname{PRF}\left(1-\alpha_{\mathrm{c}}\right) \leq \operatorname{SR} \leq \operatorname{PRF}\left(1+\alpha_{\mathrm{c}}\right)$, as indicated by the dotted and dashed lines in Fig. 7(a). In Fig. 7(c) the corresponding charge spikes are shown. For pulse repetition frequencies lower than $\mathrm{PRF}_{\min }(<100 \mathrm{~Hz})$, multiple spikes
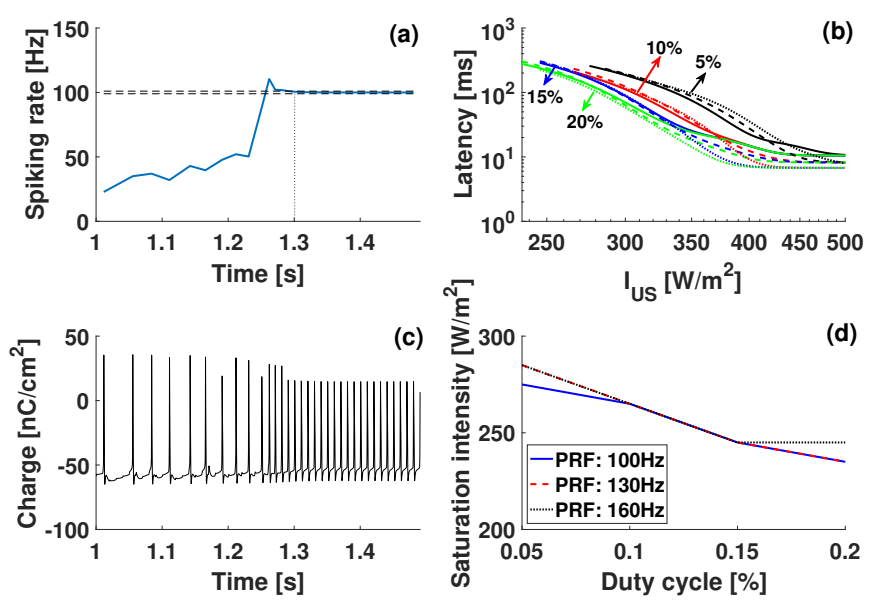

Fig. 7 Firing rate saturates to the PRF of the pulsed ultrasonic wave. (a) Spiking rate for pulsed ultrasonic stimulation ( $\mathrm{DC}=$ $10 \%, \mathrm{PRF}=100 \mathrm{~Hz}, I_{\mathrm{US}}=270 \mathrm{~W} / \mathrm{m}^{2}$ ). Horizontal dashed lines indicate the $\operatorname{PRF}\left(1 \pm \alpha_{\mathrm{c}}\right)$ values. The determination of the latency is indicated by the vertical dotted line. (b) Latency until spike saturation as function of ultrasonic intensity. Duty cycle is indicated by the arrows. (c) Membrane charge plot corresponding with the ultrasound parameters used in (a). (d) Saturation intensity versus duty cycle. Legend ((b),(d), PRF): (-) $100 \mathrm{~Hz},(--) 130 \mathrm{~Hz},(\cdots \cdots) 160 \mathrm{~Hz}$. Method: convergence factor is $\alpha_{\mathrm{c}}=1 \%$, latencies are smoothed with gaussian kernel (width $\sigma_{\mathrm{w}}=25 \mathrm{~W} / \mathrm{m}^{2}$ )

will occur during the interpulse interval and the spiking rate will not saturate reliably to the pulse repetition frequency.

Fig. 7(b) illustrates how the latency decreases with ultrasonic intensity. At the highest considered ultrasonic intensity $\left(I_{\mathrm{US}}=500 \mathrm{~W} / \mathrm{m}^{2}\right)$, the latency is close to the interpulse interval $\left(\mathrm{PRF}^{-1}\right)$. Furthermore, latencies are inversely proportional with the duty cycle, if the pulse repetion frequency is held constant. Finally, in Fig. 7(d) it is shown that the saturation intensity $I_{\text {sat }}$ is inversely proportional with the duty cycle as well.

\section{DISCUSSION}

In this study, a computational model of the plateau-potential generating subthalamic nucleus neuron (Otsuka-model, [26]) is combined with the bilayer sonophore model for ultrasoundmembrane interaction (BLS-model) [29]. The ultrasoundneuron interaction is described with the neuronal intramembrane cavitation excitation (NICE) model [28]-[30]. In the NICE-model, oscillating intraleaflet cavitations are assumed to be the main mechanism of ultrasonically induced neuron excitation. Other potential explanations of the mechanism of ultrasonic neurostimulation include flexoelectricity (Petrovmodel) [35], tension-dependent voltage-gated channels [36], and the Heimburg-Jackson soliton model [37]. In this paper, several distinctive qualitative and quantative consequences of the application of the NICE-model to the subthalamic nucleus are presented. E.g., continuous-wave insonication results in different intensity-dependent firing modes (low frequency 
repetitive spiking with negligible adaptation, high frequency spiking with significant frequency and amplitude adaptation, silenced plateaus) and pulsed excitation with sufficient repetition frequency and intensity will entrain subthalamic nucleus neurons with small latencies. Consequentially, comparison of these computational results with future experimental studies on subthalamic nucleus insonication, would help to elucidate the mechanism of ultrasonic neuromodulation.

Some limitations with respect to the Otsuka-model should be taken into account. First, the Otsuka-STN model is not physiologically complete, as currents have been omitted that have no clear relationship with the capability of subthalamic nucleus neurons to generate voltage-dependent plateaupotentials [31], [38]-[41]. These include a persistent TTXsensitive sodium current $I_{\mathrm{NaP}}$, a hyperpolarization-activated inward current $I_{\mathrm{h}}$ and high-threshold $\mathrm{Ca}^{2+}$-currents. Second, the Otsuka-model is a single compartment model and as a result neglects the spatial extent of the STN-neurons.

An alternative single compartment subthalamic nucleus model is the Rubin-Terman model [42]-[44]. However, in contrast with the Rubin-Terman model, the Otsuka-model is developed to reproduce the capability of subthalamic nucleus neurons to generate voltage-dependent plateau-potentials. Furthermore, the shape of the action potential, the dependency of the duration of the rebound response on the shape of the current pulse and the decrease in the spiking rate during a rebound burst, is simulated more reliably with the Otsukamodel [31].

A multi-compartment model of the subthalamic nucleus exists as well (Gillies-Willshaw model) [45]. However, we opted for the Otsuka-model, because of the high computational demands of the BLS-STN model (short time steps are required due to the small ultrasonic periods) and because the relatively large set of parameters in the Gillies-Willshaw model might complicate the analysis. Furthermore, as future work we aim to investigate the capabilities of ultrasonic stimulation, with a computational model of the basal ganglia network. The Otsuka-model is interesting in this context, because it has been used in computational studies on the impact of electrical deep brain stimulation on the basal ganglia network [32], [46], [47].

Another important consideration is the impact of neuronal diversity within the STN on the response to insonication and its implications for the tentative therapeutic effects of USSTN stimulation. First, variety in the electrophysiological and dynamical parameters of the plateau-potential generating STN neuron is likely to influence quantitative modeling results, e.g. lower and upper intensity thresholds ( $I_{\mathrm{tr}}^{\mathrm{l}}$ and $I_{\mathrm{tr}}^{\mathrm{u}}$, respectively). Consequentially, our results suggest that during continuouswave insonication, different plateau-potential generating STNneurons will exhibit different spiking rhythms (low frequency spiking, high frequency spiking or a silenced plateau). Furthermore, computational results indicate a narrow intensity range corresponding with the high frequency spiking mode $\left(115 \mathrm{~W} / \mathrm{m}^{2} \leq I_{\mathrm{US}} \leq 126 \mathrm{~W} / \mathrm{m}^{2}\right)$. This fact will further contribute to the diverse response to continuous-wave insonication, as practical considerations (phase aberration, variable attenuation...) will limit the possibility of achieving this level of precisely controlled ultrasound in vivo at the subthalamic nucleus. This diversity in the neuronal response would be therapeutically interesting, as a similar heterogeneous response, consisting of spiking and silenced neurons, is likely to play an important role in the mechanism of conventional electrical deep brain stimulation as well [48], [49]. Furthermore, assuming that qualitative results are robust to this neuronal diversity, our results indicate that a homogeneous high-frequency spiking response in the STN will be induced by pulsed ultrasonic stimulation. Second, more profound sources of STN-diversity are GABAergic interneurons [50] and neurons incapable of generating plateau-potentials [25], [38]. Because these sources will interact with the response to ultrasound of the plateaupotential generating STN neurons, we believe that this topic is best addressed as future work within the context of a computational model, coupling ultrasound with the basal ganglia network.

Finally, the reported computational simulations of STN insonication predict that relatively low intensity levels are required to elicit high frequency spiking or silenced plateaupotentials, compared to previous modeling studies for cortical insonication [29], [30] and experimental studies [23]. We attribute these significantly lower required ultrasonic intensities to the different membrane channel dynamics of the STN. E.g., STN-neurons are spontaneously active and are able to generate voltage-dependent plateau-potentials. The former STN-property (spontaneous firing) corresponds with the low required intensities to induce high-frequency spiking, while the latter property (plateau-potentials) is associated with the induction of a silenced plateau at higher, but still relatively low, intensities. This prediction that lower intensities are required in order to modulate STN-activity, compared to cortical stimulation, contributes to the theoretical feasibility of safe lowintensity ultrasonic deep brain modulation without activating overlying cortical areas.

\section{CONCLUSION}

In this study, a computational model for ultrasonic STNstimulation is constructed, by combining the Otsuka-STN model with the bilayer sonophore model for ultrasoundmembrane interaction. The model implementation was verified by a careful comparison of simulation results with literature for two restricted cases of the STN-BLS model: electrical stimulation of the STN and ultrasonic stimulation of cortical neurons (STN is substituted by RS, FS or LTS neurons). Furthermore, a convergence analysis was performed on the discretisation parameters (maximal timestep, tolerances, update time, table resolutions,... ) to ensure that the model results are not significantly dependent on the implementation.

This research indicates that ultrasonic neuromodulation might be promising in the context of non-invasive STN-DBS. While pulsed or intermediate intensity continuous-wave stimulation results in high-frequency firing of the STN-model, high intensity continuous-wave insonication is able to silence the STN at an elevated plateau. The former is similar to electrical deep brain stimulation, while the latter might have similar functionality as subthalamotomy. The intensity thresholds for continuous-wave stimulation that delineate the transition from 
high frequency firing to a silenced plateau are increasing with ultrasonic driving frequency. The predicted ultrasonic intensity thresholds are relatively low, implying that low-intensity noninvasive ultrasonic deep brain stimulation without excitation of the overlying cortical areas is feasible. Furthermore, pulsed ultrasonic stimulation is able to modulate spiking rates with small latencies (close to $\mathrm{PRF}^{-1}$ for $I_{\mathrm{US}}=500 \mathrm{~W} / \mathrm{m}^{2}$, latency inversely proportional to $\mathrm{DC}$ and $I_{\mathrm{US}}$ ).

The theoretical capability of ultrasound to differentially silence or excite subthalamic nucleus neurons non-invasively and with millimeter precision, makes acoustic neuromodulation interesting as a tool to study basal ganglia function and the mechanism of deep brain stimulation in the treatment of movement disorders. The authors intend to use this study as a basis for future research, by incorporating the BLS-STN model in a computational network model of the cortex-basal ganglia-thalamus system. The goal is to examine the capability of ultrasound to modulate pathological beta-oscillations and hypersynchrony in the Parkinsonian basal ganglia.

\section{CONFLICT OF INTERESTS}

The authors declare that there is no conflict of interest regarding the publication of this paper.

\section{APPENDIX}

\section{SUMMARY OF MODEL EQUATIONS}

This appendix complements the method section, by including all model equations and parameter values that are necessary to reproduce the results obtained in this paper. A summary of the general parameter values is given in Table I

TABLE I Summary of General Model Parameters

\begin{tabular}{|c|c|c|}
\hline Abbr. & Value & Description \\
\hline$T$ & $33^{\circ} \mathrm{C}$ & Temperature \\
\hline$R_{\mathrm{g}}$ & $8.314 \mathrm{~J} /(\mathrm{K} \cdot \mathrm{mol})$ & Universal gas constant \\
\hline$F^{\circ}$ & $96485 \mathrm{C} / \mathrm{mol}$ & Faraday constant \\
\hline$\epsilon_{0}$ & $8.85 \cdot 10^{-12} \mathrm{~F} / \mathrm{m}$ & Absolute vacuumpermittivity \\
\hline$\epsilon_{\mathrm{r}}$ & 1 & Relative vacuumpermittivity \\
\hline$c$ & $1515 \mathrm{~m} / \mathrm{s}$ & Speed of sound in CSF \\
\hline$\rho_{1}$ & $1028 \mathrm{~kg} / \mathrm{m}^{3}$ & Density of CSF \\
\hline$k_{\mathrm{a}}$ & $1.63 \cdot 10^{5} \mathrm{~Pa} \cdot \mathrm{m}^{3} / \mathrm{mol}$ & Henry constant (air in CSF) \\
\hline$D_{\mathrm{a}}$ & $3 \cdot 10^{-9} \mathrm{~m}^{2} / \mathrm{s}$ & Diffusion coefficient (air in CSF) \\
\hline$k_{\mathrm{S}}$ & $0.24 \mathrm{~N} / \mathrm{m}$ & Areal modulus \\
\hline$\delta_{0}$ & $2 \mathrm{~nm}$ & Thickness leaflet \\
\hline$\mu_{\mathrm{s}}$ & $0.035 \mathrm{~Pa} \cdot \mathrm{s}$ & Dynamic viscosity of leaflets \\
\hline$\mu_{1}$ & $0.7 \cdot 10^{-3} \mathrm{~Pa} \cdot \mathrm{s}$ & Dynamic viscosity of the CSF \\
\hline
\end{tabular}

\section{A. Subthalamic nucleus model}

The subthalamic nucleus model is adopted from Otsuka et al. [26], [31]. The membrane currents are modeled by:

$$
\begin{gathered}
I_{\mathrm{Na}}=g_{\mathrm{Na}} m^{3} h\left(V-V_{\mathrm{Na}}\right), \\
I_{\mathrm{K}}=g_{\mathrm{K}} n^{4}\left(V-V_{\mathrm{K}}\right), \\
I_{\mathrm{A}}=g_{\mathrm{A}} a^{2} b\left(V-V_{\mathrm{K}}\right), \\
I_{\mathrm{T}}=g_{\mathrm{T}} p^{2} q\left(V-V_{\mathrm{Ca}}\right), \\
I_{\mathrm{L}}=g_{\mathrm{L}} c^{2} d_{1} d_{2}\left(V-V_{\mathrm{Ca}}\right),
\end{gathered}
$$

TABLE II Summary of Membrane Current Model Parameters

\begin{tabular}{|c|c|c|}
\hline Membrane current & $g\left[\mathrm{mS} / \mathrm{cm}^{2}\right]$ & $V_{\mathrm{r}}[\mathrm{mV}]$ \\
\hline$I_{\mathrm{Na}}$ & 49 & 60 \\
$I_{\mathrm{K}}$ & 57 & -90 \\
$I_{\mathrm{A}}$ & 5 & -90 \\
$I_{\mathrm{T}}$ & 5 & $\frac{R_{\mathrm{g} T}}{2 F} \ln \frac{C a_{0}}{C a_{\mathrm{i}}}$ \\
$I_{\mathrm{L}}$ & 15 & $\frac{R_{\mathrm{g} T}}{2 F} \ln \frac{C a_{0}}{C a_{\mathrm{i}}}$ \\
$I_{\mathrm{CaK}}$ & 1 & -90 \\
$I_{1}$ & 0.35 & -60 \\
\hline
\end{tabular}

The calcium reversal potential is calculated by the Nernst-equation $\left(C a_{0}=2 \mathrm{mM}\right)$

$$
\begin{gathered}
I_{\mathrm{CaK}}=g_{\mathrm{CaK}} r^{2}\left(V-V_{\mathrm{K}}\right), \\
I_{1}=g_{1}\left(V-V_{1}\right) .
\end{gathered}
$$

The membrane conductance and reversal potential for the different membrane currents are summarized in Table [I

The dynamics of the gate parameters is given by:

$$
\frac{\mathrm{d} X}{\mathrm{~d} t}=\frac{X_{\infty}-X}{\tau_{X}} .
$$

$X_{\infty}$ is a function of $V_{\mathrm{m}}$, if $X$ represents $m, h, n, p, q, a, b$, $c$ or $d_{1}$ :

$$
X_{\infty}=\frac{1}{1+\exp \left[\frac{V_{\mathrm{m}}-\theta_{X}}{k_{X}}\right]} .
$$

Conversely, $X_{\infty}$ is a function of $C a_{\mathrm{i}}$ if $X$ represents $d_{2}$ or $r$. In this case, the membrane potential $V_{\mathrm{m}}$ in eq. (14) is replaced by the intracellullar calcium concentration $C a_{\mathrm{i}}$.

Similarly, general expressions are used for the time constant $\tau_{X}$. If $X$ represents $m$ or $a$ :

$$
\tau_{X}=\tau_{0}+\frac{\tau_{1}}{1+\exp \left(\frac{-\left[V_{\mathrm{m}}-\theta_{\tau}\right]}{\sigma_{\tau}}\right)} .
$$

And for $X=h, n, p, q, b, c, d_{1}$ :

$$
\tau_{X}=\tau_{0}+\frac{\tau_{1}}{\exp \left(\frac{-\left[V_{\mathrm{m}}-\theta_{\tau 1}\right]}{\sigma_{\tau 1}}\right)+\exp \left(\frac{-\left[V_{\mathrm{m}}-\theta_{\tau 2}\right]}{\sigma_{\tau 2}}\right)} .
$$

Finally, for calcium dynamics we used the time constants: $\tau_{\mathrm{r}}=2 \mathrm{~ms}$ and $\tau_{d_{2}}=130 \mathrm{~ms}$. The model constants for the membrane gate dynamics are summarized in Table III

\section{B. Bilayer sonophore model}

The bilayer sonophore model equations and parameter values are adopted from [28]-[30]. At $t=0 \mathrm{~ms}$, the leaflet is at equilibrium (i.e., $Z=0 \mathrm{~nm}$ and $\frac{\mathrm{d} Z}{\mathrm{~d} t}=0 \mathrm{~nm} / \mathrm{s}$ ) and a quasistatic approximation can be used to calculate the dynamics of the membrane leaflet:

$$
P_{\mathrm{in}}^{\mathrm{QS}}+P_{\mathrm{M}}+P_{\mathrm{A}} \sin (\omega t)+P_{\mathrm{ec}}-P_{0}=0 .
$$

Here, $P_{\mathrm{in}}^{\mathrm{QS}}$ is a quasi-static approximation of the internal pressure:

$$
P_{\mathrm{in}}^{\mathrm{QS}}=P_{0} \frac{\pi a^{2} \Delta}{V_{\mathrm{a}}(Z)^{\kappa}}
$$

The polytropic constant $\kappa$ is assumed to be unity (small volume of air in the intraleaflet cavity). Eventually, if 
$Z \geq Z_{\mathrm{QS}}$ for some small value of $Z_{\mathrm{QS}}$, the mechanics of the leaflet and cerebrospinal fluid (CSF) has to be taken into account and the quasi-static equation is replaced by an adaptation of the Rayleigh-Plesset equation.

The internal gas pressure $P_{\text {in }}$ in the Rayleigh-Plesset equation is calculated by the universal gas law:

$$
P_{\text {in }}=\frac{n_{\mathrm{a}} R_{\mathrm{g}} T}{V_{\mathrm{a}}(Z)} .
$$

Here, the mole of air $n_{\mathrm{a}}$ inside the intraleaflet cavity, is calculated by a diffusion equation:

$$
\frac{\mathrm{d} n_{\mathrm{a}}}{\mathrm{d} t}=\frac{2 S(Z) D_{\mathrm{a}}}{\xi}\left[C_{\mathrm{a}}-P_{\mathrm{in}} / k_{\mathrm{a}}\right] .
$$

$S(Z)$ is the area of a single leaflet, $D_{\mathrm{a}}$ is the diffusion coefficient of air, $\xi=0.5 \mathrm{~nm}$ is an effective length of the boundary layer for air diffusion, $k_{\mathrm{a}}$ is the Henry-coefficient of dissolved air in CSF, and $C_{\mathrm{a}}$ is the molar air concentration in the CSF. A more general simulation of the diffusion of air in the environment of an oscillating intraleaflet cavity is performed in [28] (Fick's law). However, as noted in [29], the simplification in eq. 20] is justified and is computationally more efficient.

The molecular pressure term $P_{\mathrm{M}}$ is calculated by [29]:

$$
P_{\mathrm{M}}=A_{\mathrm{r}} \frac{2 \pi}{S(Z)} \int_{0}^{a} r \mathrm{~d} r\left[\left(\frac{\Delta^{*}}{2 z(r)+\Delta}\right)^{x}-\left(\frac{\Delta^{*}}{2 z(r)+\Delta}\right)^{y}\right] .
$$

Here, $A_{\mathrm{r}}=10^{5} \mathrm{~Pa}$ is the attraction/repulsion pressure coefficient, $x=5$ and $y=3.3$ are the repulsion and attraction exponents, respectively, and $\Delta^{*}=1.4 \mathrm{~nm}$ is the initial intraleaflet gap if no membrane charge would be present. The local leaflet displacement $z(r)$ is calculated by:

$$
z(r)=\operatorname{sign}(R(Z)) \sqrt{R(Z)^{2}-r^{2}}-R(Z)+Z .
$$

The electrostatic pressure $P_{\mathrm{ec}}$ and the pressure due to the leaflet tension $P_{\mathrm{S}}$ are given by:

$$
\begin{gathered}
P_{\mathrm{ec}}=-\frac{\pi a^{2}}{S(Z)} \frac{Q_{\mathrm{m}}^{2}}{2 \epsilon_{\mathrm{r}} \epsilon_{0}}, \\
P_{\mathrm{S}}(Z)=\frac{2 k_{\mathrm{s}} Z^{3}}{a^{2}\left(a^{2}+Z^{2}\right)} .
\end{gathered}
$$

Here, $Q_{\mathrm{m}}=C_{\mathrm{m}} V_{\mathrm{m}}$ is the membrane charge and $k_{s}$ is the areal modulus of the bilayer membrane. Finally, a static pressure of $P_{0}=10^{5} \mathrm{~Pa}$ is used.

In the bilayer sonophore model equations above, the leaflet surface and intraleaflet cavity volume are functions of the displacement $Z$ :

$$
\begin{gathered}
S(Z)=\pi\left(a^{2}+Z^{2}\right), \\
V_{\mathrm{a}}(Z)=\pi a^{2} \Delta\left[1+Z /(3 \Delta)\left(\frac{Z^{2}}{a^{2}}+3\right)\right] .
\end{gathered}
$$

TABLE III Model Parameters for the Gate Dynamics

\begin{tabular}{|c|c|c|c|c|c|}
\hline$X$ & $\theta_{X}[\mathrm{mV}]^{a}$ & $k_{X}[\mathrm{mV}]^{a}$ & $\tau_{0}, \tau_{1}[\mathrm{~ms}]$ & $\theta_{\tau}[\mathrm{mV}]^{b}$ & $\sigma_{\tau}[\mathrm{mV}]^{b}$ \\
\hline$a$ & -45 & -14.7 & 1,1 & -40 & -0.5 \\
$b$ & -90 & 7.5 & 0,200 & $-60,-40$ & $-30,-10$ \\
$c$ & -30.6 & -5 & 45,10 & $-27,-50$ & $-20,15$ \\
$d_{1}$ & -60 & 7.5 & 400,500 & $-40,-20$ & $-15,20$ \\
$d_{2}$ & 0.1 & 0.02 & 130 & - & - \\
$m$ & -40 & -8 & $0.2,3$ & -53 & -0.7 \\
$h$ & -45.5 & 6.4 & $0,24.5$ & $-50,-50$ & $-15,16$ \\
$n$ & -41 & -14 & 0,11 & $-40,-40$ & $-40,50$ \\
$p$ & -56 & -6.7 & $5,0.33$ & $-27,-102$ & $-10,15$ \\
$q$ & -85 & 5.8 & 0,400 & $-50,-50$ & $-15,16$ \\
$r$ & 0.17 & -0.08 & 2 & - & - \\
\hline
\end{tabular}

(a) The calcium gate constants $\theta_{d_{2}}, \theta_{r}, k_{d_{2}}$, and $k_{r}$ are expressed in $[\mu \mathrm{M}]$ instead of $[\mathrm{mV}]$. (b) If two values are tabulated, then they refer to $\theta_{\tau 1}$ and $\theta_{\tau 2}$ or $\sigma_{\tau 1}$ and $\sigma_{\tau 2}$, respectively.

\section{REFERENCES}

[1] V. K. Khanna, "Deep brain stimulation," in Implantable Medical Electronics. Springer, 2016, pp. 309-329.

[2] K. Sugiyama, "Complications of deep brain stimulation," in Deep Brain Stimulation for Neurological Disorders. Springer, 2015, pp. 195-206.

[3] A. L. Benabid, S. Chabardes, J. Mitrofanis, and P. Pollak, "Deep brain stimulation of the subthalamic nucleus for the treatment of parkinson's disease," The Lancet Neurology, vol. 8, no. 1, pp. 67-81, 2009.

[4] N. Grossman, D. Bono, N. Dedic, S. B. Kodandaramaiah, A. Rudenko, H.-J. Suk, A. M. Cassara, E. Neufeld, N. Kuster, L.-H. Tsai et al., "Noninvasive deep brain stimulation via temporally interfering electric fields," Cell, vol. 169, no. 6, pp. 1029-1041, 2017.

[5] A. Gunduz and M. S. Okun, "A new non-surgical approach for deepbrain stimulation," The Lancet Neurology, vol. 16, no. 8, p. e1, 2017.

[6] R. Chen, G. Romero, M. G. Christiansen, A. Mohr, and P. Anikeeva, "Wireless magnetothermal deep brain stimulation," Science, vol. 347 , no. 6229, pp. 1477-1480, 2015.

[7] M. Pernot, J.-F. Aubry, M. Tanter, J.-L. Thomas, and M. Fink, "High power transcranial beam steering for ultrasonic brain therapy," Physics in medicine and biology, vol. 48, no. 16, p. 2577, 2003.

[8] G. T. Clement, P. J. White, R. L. King, N. McDannold, and K. Hynynen, "A magnetic resonance imaging-compatible, large-scale array for transskull ultrasound surgery and therapy," Journal of ultrasound in medicine, vol. 24, no. 8, pp. 1117-1125, 2005.

[9] L. Marsac, D. Chauvet, B. Larrat, M. Pernot, B. Robert, M. Fink, A.L. Boch, J.-F. Aubry, and M. Tanter, "Mr-guided adaptive focusing of therapeutic ultrasound beams in the human head," Medical physics, vol. 39, no. 2, pp. 1141-1149, 2012.

[10] D. Coluccia, J. Fandino, L. Schwyzer, R. OGorman, L. Remonda, J. Anon, E. Martin, and B. Werner, "First noninvasive thermal ablation of a brain tumor with mr-guided focusedultrasound," Journal of therapeutic ultrasound, vol. 2, no. 1, p. 17, 2014.

[11] R. L. King, J. R. Brown, W. T. Newsome, and K. B. Pauly, "Effective parameters for ultrasound-induced in vivo neurostimulation," Ultrasound in medicine \& biology, vol. 39, no. 2, pp. 312-331, 2013.

[12] R. L. King, J. R. Brown, and K. B. Pauly, "Localization of ultrasoundinduced in vivo neurostimulation in the mouse model," Ultrasound in medicine \& biology, vol. 40, no. 7, pp. 1512-1522, 2014.

[13] E. N. Harvey, "The effect of high frequency sound waves on heart muscle and other irritable tissues," American Journal of PhysiologyLegacy Content, vol. 91, no. 1, pp. 284-290, 1929.

[14] F. Fry et al., "Production of reversible changes in the central nervous system by ultrasound," Science, vol. 127, pp. 83-84, 1958.

[15] W. J. Tyler, Y. Tufail, M. Finsterwald, M. L. Tauchmann, E. J. Olson, and C. Majestic, "Remote excitation of neuronal circuits using low-intensity, low-frequency ultrasound," PloS one, vol. 3, no. 10, p. e3511, 2008.

[16] Y. Tufail, A. Matyushov, N. Baldwin, M. L. Tauchmann, J. Georges, A. Yoshihiro, S. I. H. Tillery, and W. J. Tyler, "Transcranial pulsed ultrasound stimulates intact brain circuits," Neuron, vol. 66, no. 5, pp. 681-694, 2010.

[17] H. Kim, S. J. Taghados, K. Fischer, L.-S. Maeng, S. Park, and S.-S. Yoo, "Noninvasive transcranial stimulation of rat abducens nerve by focused ultrasound," Ultrasound in medicine \& biology, vol. 38, no. 9, pp. 1568-1575, 2012. 
[18] H. Kim, A. Chiu, S. D. Lee, K. Fischer, and S.-S. Yoo, "Focused ultrasound-mediated non-invasive brain stimulation: examination of sonication parameters," Brain stimulation, vol. 7, no. 5, pp. 748-756, 2014.

[19] H. Kim, M. Y. Park, S. D. Lee, W. Lee, A. Chiu, and S.-S. Yoo, "Suppression of eeg visual-evoked potentials in rats via neuromodulatory focused ultrasound," Neuroreport, vol. 26, no. 4, p. 211, 2015.

[20] W. Legon, T. F. Sato, A. Opitz, J. Mueller, A. Barbour, A. Williams, and W. J. Tyler, "Transcranial focused ultrasound modulates the activity of primary somatosensory cortex in humans," Nature neuroscience, vol. 17, no. 2, p. 322, 2014.

[21] W. Lee, H.-C. Kim, Y. Jung, Y. A. Chung, I.-U. Song, J.-H. Lee, and S.S. Yoo, "Transcranial focused ultrasound stimulation of human primary visual cortex," Scientific reports, vol. 6, p. 34026, 2016.

[22] O. Naor, S. Krupa, and S. Shoham, "Ultrasonic neuromodulation," Journal of neural engineering, vol. 13, no. 3, p. 031003, 2016.

[23] H. Baek, K. J. Pahk, and H. Kim, "A review of low-intensity focused ultrasound for neuromodulation," Biomedical Engineering Letters, pp. $1-8,2017$.

[24] H. Nakanishi, H. Kita, and S. Kitai, "Electrical membrane properties of rat subthalamic neurons in an in vitro slice preparation," Brain research, vol. 437, no. 1, pp. 35-44, 1987.

[25] T. Otsuka, F. Murakami, and W.-J. Song, "Excitatory postsynaptic potentials trigger a plateau potential in rat subthalamic neurons at hyperpolarized states," Journal of Neurophysiology, vol. 86, no. 4, pp. $1816-1825,2001$.

[26] T. Otsuka, T. Abe, T. Tsukagawa, and W.-J. Song, "Conductance-based model of the voltage-dependent generation of a plateau potential in subthalamic neurons," Journal of neurophysiology, vol. 92, no. 1, pp. 255-264, 2004.

[27] J. Baufreton, M. Garret, A. Rivera, A. de la Calle, F. Gonon, B. Dufy, B. Bioulac, and A. Taupignon, "D5 (not d1) dopamine receptors potentiate burst-firing in neurons of the subthalamic nucleus by modulating an 1-type calcium conductance," Journal of Neuroscience, vol. 23, no. 3 , pp. 816-825, 2003.

[28] B. Krasovitski, V. Frenkel, S. Shoham, and E. Kimmel, "Intramembrane cavitation as a unifying mechanism for ultrasound-induced bioeffects," Proceedings of the National Academy of Sciences, vol. 108, no. 8, pp. 3258-3263, 2011.

[29] M. Plaksin, S. Shoham, and E. Kimmel, "Intramembrane cavitation as a predictive bio-piezoelectric mechanism for ultrasonic brain stimulation," Physical review X, vol. 4, no. 1, p. 011004, 2014.

[30] M. Plaksin, E. Kimmel, and S. Shoham, "Cell-type-selective effects of intramembrane cavitation as a unifying theoretical framework for ultrasonic neuromodulation," eNeuro, vol. 3, no. 3, pp. ENEURO-0136, 2016.

[31] T. Heida, E. Marani, and K. G. Usunoff, The subthalamic nucleus: Part II: Modelling and simulation of activity. Springer Science \& Business Media, 2008, vol. 199.

[32] P. J. Hahn and C. C. McIntyre, "Modeling shifts in the rate and pattern of subthalamopallidal network activity during deep brain stimulation," Journal of computational neuroscience, vol. 28, no. 3, pp. 425-441, 2010.

[33] L. F. Shampine and M. W. Reichelt, "The matlab ode suite," SIAM journal on scientific computing, vol. 18, no. 1, pp. 1-22, 1997.

[34] R. Ashino, M. Nagase, and R. Vaillancourt, "Behind and beyond the matlab ode suite," Computers \& Mathematics with Applications, vol. 40, no. 4, pp. 491-512, 2000

[35] A. G. Petrov, "Flexoelectricity of model and living membranes," Biochimica et Biophysica Acta (BBA)-Biomembranes, vol. 1561, no. 1, pp. 1-25, 2002.

[36] C. E. Morris and P. F. Juranka, "Nav channel mechanosensitivity: activation and inactivation accelerate reversibly with stretch," Biophysical Journal, vol. 93, no. 3, pp. 822-833, 2007.

[37] T. Heimburg and A. D. Jackson, "On soliton propagation in biomembranes and nerves," Proceedings of the National Academy of Sciences of the United States of America, vol. 102, no. 28, pp. 9790-9795, 2005.

[38] C. Beurrier, P. Congar, B. Bioulac, and C. Hammond, "Subthalamic nucleus neurons switch from single-spike activity to burst-firing mode," Journal of Neuroscience, vol. 19, no. 2, pp. 599-609, 1999.

[39] C. Beurrier, B. Bioulac, and C. Hammond, "Slowly inactivating sodium current (i nap) underlies single-spike activity in rat subthalamic neurons," Journal of Neurophysiology, vol. 83, no. 4, pp. 1951-1957, 2000.

[40] M. D. Bevan and C. J. Wilson, "Mechanisms underlying spontaneous oscillation and rhythmic firing in rat subthalamic neurons," Journal of Neuroscience, vol. 19, no. 17, pp. 7617-7628, 1999.
[41] W.-J. Song, Y. Baba, T. Otsuka, and F. Murakami, "Characterization of ca 2+ channels in rat subthalamic nucleus neurons," Journal of Neurophysiology, vol. 84, no. 5, pp. 2630-2637, 2000.

[42] D. Terman, J. E. Rubin, A. Yew, and C. Wilson, "Activity patterns in a model for the subthalamopallidal network of the basal ganglia," Journal of Neuroscience, vol. 22, no. 7, pp. 2963-2976, 2002.

[43] J. E. Rubin and D. Terman, "High frequency stimulation of the subthalamic nucleus eliminates pathological thalamic rhythmicity in a computational model," Journal of computational neuroscience, vol. 16, no. 3, pp. 211-235, 2004

[44] R. Q. So, A. R. Kent, and W. M. Grill, "Relative contributions of local cell and passing fiber activation and silencing to changes in thalamic fidelity during deep brain stimulation and lesioning: a computational modeling study," Journal of computational neuroscience, vol. 32, no. 3 , pp. 499-519, 2012.

[45] A. Gillies and D. Willshaw, "Membrane channel interactions underlying rat subthalamic projection neuron rhythmic and bursting activity," Journal of neurophysiology, vol. 95, no. 4, pp. 2352-2365, 2006.

[46] K. Kumaravelu, D. T. Brocker, and W. M. Grill, "A biophysical model of the cortex-basal ganglia-thalamus network in the 6-ohda lesioned rat model of parkinsons disease," Journal of computational neuroscience, vol. 40, no. 2, pp. 207-229, 2016.

[47] G. Kang and M. M. Lowery, "Interaction of oscillations, and their suppression via deep brain stimulation, in a model of the corticobasal ganglia network," IEEE Transactions on Neural Systems and Rehabilitation Engineering, vol. 21, no. 2, pp. 244-253, 2013.

[48] S. Bellinger, G. Miyazawa, and P. Steinmetz, "Submyelin potassium accumulation may functionally block subsets of local axons during deep brain stimulation: a modeling study," Journal of neural engineering, vol. 5 , no. 3, p. 263,2008

[49] M. D. Humphries and K. Gurney, "Network effects of subthalamic deep brain stimulation drive a unique mixture of responses in basal ganglia output," European journal of neuroscience, vol. 36, no. 2, pp. 2240$2251,2012$.

[50] J.-C. Lévesque and A. Parent, "Gabaergic interneurons in human subthalamic nucleus," Movement disorders: official journal of the Movement Disorder Society, vol. 20, no. 5, pp. 574-584, 2005. 